Medicine, Bristol University, Bristol, UK; ${ }^{3}$ Gastroenterology Unit, Ospedale Valduce, Como, Italy

Introduction Although current guidelines recommend Small-Bowel Capsule Endoscopy (SBCE) for the evaluation of patients with obscure gastrointestinal bleeding (OGIB), its role in investigating patients with iron deficiency anaemia (IDA) alone is still unclear. Aim To evaluate the diagnostic yield (DY) of SBCE in patients with IDA by means of a systematic review of studies reporting this data. Methods A recursive literature search (sources: Medline and Embase) of studies reporting DY of SBCE in patients with IDA was undertaken (November 2011). The search was restricted to fully reported papers in English, published between January 2001 and November 2011, including adult patients and clearly reporting DY for IDA patients. Studies were selected and evaluated separately by two of the authors. Data on DY were extracted, pooled, and analysed. Any discrepancy in papers selection or in data extraction was solved by consensus. The QUADAS tool was used to assess the study for methodological quality. Statistical analysis was performed with STATA version 12.0 .

Results A total of 27 (7 prospective, 20 retrospective; total of 1943 patients) studies was selected for final review and analysis. Five studies (including 316 patients) were specifically designed to evaluate only IDA patients; in the remaining 22 studies, the patients with IDA represented a subgroup of patients undergoing SBCE. Overall, the 27 studies were of poor to moderate quality. The overall pooled DY, estimated by applying the random effect model $\left(\mathrm{I}^{2}\right.$ : $77.7 \%$ ), was $48.2 \%$ (95\% CI 43.1 to $53.4 \%$ ) while it was $53.4 \%$ (95\% CI 34.7 to $72.0 \%$ ) and $47.2 \%$ (95\% CI 42.1 to $52.2 \%$ ) for studies focusing and not focusing on IDA patients, respectively.

Conclusion Although the studies evaluating the DY of SBCE in IDA are of poor to moderate quality and heterogeneous, the estimated DY is about $50 \%$ and seems to be comparable with that observed in patients with OBIG.

Competing interests None declared.

\section{PTU-146 DUAL ENERGY X-RAY ABSORPTIOMETRY (DEXA) SCANS IN COELIAC DISEASE (CD): ARE BSG GUIDELINES FOLLOWED?}

doi:10.1136/gutjnl-2012-302514c.146

B Krishnan, ${ }^{*}$ V Sehgal, D Ramdass, K Besherdas. Chasefarm District General Hospital, London, UK

Introduction Patients with CD have a higher incidence of osteopenia or osteoporosis due to reduced bone mineral density (BMD). However, significant improvement in $\mathrm{BMD}$ and calcium absorption is seen after introduction of gluten-free diet (GFD). The BSG recommend (with poor evidence) that DEXA scan should only be performed after introduction of GFD in patients with high risk (two or more of the following: age $>70$, low BMI, weight loss more than $10 \mathrm{~kg}$ and those with persistent symptoms on GFD for a year or non-compliance with diet). The aim of this study was to look at our practice of DEXA scanning in CD patients and assess whether BSG guidelines were followed and whether the timing of DEXA scans made any difference to outcome.

Methods We reviewed all 82 patients from our coeliac database who were diagnosed between April 1985 and November 2011 at a district general hospital in North London (Chase Farm Hospital). 14 patients were excluded from the study as medical notes were missing or they were lost to follow-up. Two patients did not have DEXA scan. Data were analysed retrospectively by review of medical and electronic records. Patient demographics, BMI, history of weight loss, age at diagnosis, date of DEXA scan and results were recorded. We used the standard WHO definition for osteoporosis
( $T$ score $<-2.5$ ), osteopenia ( $T$ score between -1 and -2.5 ) and normal (T score $>-1$ ).

Results The mean patient age was 54 (range 19-93) with 52 females. The mean time interval between diagnosis and DEXA was 2 years and 10 months (range 0 month-33 years) with a median of 8 months. 37 patients (56\%) had DEXA scan within a year of diagnosis of which 16 (43\%) were normal, and the rest had osteopenia $(24 \%)$ or osteoporosis (32\%). Of the remaining patients $(45 \%)$, nine had normal DEXA, five had osteopenia and 11 had osteoporosis. Comparing these two groups of patients the timing of DEXA scan (1 year of diagnosis) was not statistically significant in terms of outcome ( $\mathrm{p}$ value-1.0000). However $80 \%$ of patients over the age of 70 years had osteoporosis. There was no record of BMI, history of weight loss or other risk factors for osteoporosis prior to DEXA request.

Conclusion Our practice of DEXA scan did not adhere to the BSG guidelines. There was great variability in timing of DEXA scans in $\mathrm{CD}$ patients. There was marked absence of record keeping in terms of BMI, history of weight loss and other risk factors to guide DEXA requests. A large proportion of patients (80\%) with CD over age of 70 had osteoporosis. The timing of the DEXA scan did not significantly affect the T score. The lack of adherence to guidance could be because of its poor evidence base and also there is no clear recommendation on repeat DEXA scanning following initial assessment. We would recommend clearer guidance on the assessment of osteoporosis in CD.

Competing interests None declared.

\section{PTU-147 STRUCTURED GASTROENTEROLOGICAL EVALUATION AND IMPROVED OUTCOMES FOR PATIENTS WITH CHRONIC GASTROINTESTINAL SYMPTOMS FOLLOWING PELVIC RADIOTHERAPY}

doi:10.1136/gutjnl-2012-302514c.147

${ }^{1} \mathrm{C}$ C Henson, ${ }^{* 2} \mathrm{~J}$ McLaughlin, ${ }^{2} \mathrm{Y}$ Ang, ${ }^{3} \mathrm{C}$ Babbs, ${ }^{4} \mathrm{~J}$ Crampton, ${ }^{4} \mathrm{M}$ Kelly, ${ }^{3} \mathrm{~S}$ Lal, ${ }^{5} \mathrm{~J}$ Limdi, ${ }^{6} \mathrm{G}$ Whatley, ${ }^{1} \mathrm{R}$ Swindell, 'W Makin, ${ }^{1} \mathrm{~S}$ E Davidson. ${ }^{1}$ Christie Hospital, Manchester, UK; ${ }^{2}$ University of Manchester, Manchester, UK; ${ }^{3}$ Salford Royal Foundation Trust, Salford, UK; ${ }^{4}$ University Hospital of South Manchester NHS Trust, Manchester, UK; ${ }^{5}$ Pennine Acute Hospitals NHS Trust, Manchester, UK; ${ }^{6}$ Tameside Hospital, Ashton under Lyne, UK

Introduction 17000 patients are treated with radical pelvic radiotherapy per year in the UK. 50\% will develop chronic gastrointestinal (GI) symptoms that adversely affect quality-of-life, which have been shown to persist at the same level of severity for at least 3 years following treatment. Despite this, fewer than 20\% are referred to a gastroenterologist. We aimed to determine if structured gastroenterological evaluation improves symptoms this patient group.

Methods 60 patients with GI symptoms $\geq 6$ months after radical pelvic radiotherapy were identified from oncology clinics. Those requiring urgent investigation via the 2 -week wait pathway were excluded. They were assessed at baseline using patient-reported symptom-based questionnaires: inflammatory bowel disease questionnaire (IBDQ); Vaizey incontinence questionnaire (VIO); and the Common Terminology Criteria for Adverse Events (CTCAE) pelvis questionnaire. Participants were then referred to and managed by gastroenterologists using an algorithmic approach, which involves the identification of all GI symptoms and investigation for all potential causes for these symptoms. Further assessments were made at 3 and 6 months using the questionnaires.

Results 20 men and 36 women were included, with a median age of 58.5 years (range 26.9-81.8). Median time from radiotherapy to baseline gastroenterological assessment was 3.0 years (range 0.6-18.7). Median IBDO score improved from 168 at baseline to 195 